PROCEEDINGS OF THE

AMERICAN MATHEMATICAL SOCIETY

Volume 129, Number 12, Pages 3549-3557

S 0002-9939(01)06141-X

Article electronically published on May 3, 2001

\title{
STRUCTURE OF THE FIXED POINT SET AND COMMON FIXED POINTS OF ASYMPTOTICALLY NONEXPANSIVE MAPPINGS
}

\author{
T. DOMÍNGUEZ BENAVIDES AND P. LORENZO RAMÍREZ
}

(Communicated by Jonathan M. Borwein)

\begin{abstract}
Let $X$ be a Banach space, $C$ a weakly compact convex subset of $X$ and $T: C \rightarrow C$ an asymptotically nonexpansive mapping. Under the usual assumptions on $X$ which assure the existence of fixed point for $T$, we prove that the set of fixed points is a nonexpansive retract of $C$. We use this result to prove that all known theorems about existence of fixed point for asymptotically nonexpansive mappings can be extended to obtain a common fixed point for a commuting family of mappings. We also derive some results about convergence of iterates.
\end{abstract}

\section{INTRODUCTION}

One of the most celebrated results in Metric Fixed Point Theory was given by R. Bruck [1] who proved that the fixed point set of a nonexpansive mapping $T: C \rightarrow C$ is a nonexpansive retract of $C$ when $C$ is a weakly compact convex subset of a Banach space $X$ which has "nice" properties with respect to the existence of fixed point. The same author [2] used this fact to derive the existence of fixed points for a commuting family of nonexpansive mappings. In a later paper [3] he wondered about the validity of similar results for more general classes of mappings. In this paper we prove some Bruck type results for different mappings which satisfy some assumptions concerning asymptotic nonexpansiveness. If $X$ and $C$ are as above, a mapping $T: C \rightarrow C$ is said to be asymptotically nonexpansive if there exists a sequence $\left\{k_{n}\right\}$ of real numbers with $\lim _{n} k_{n}=1$ such that

$$
\left\|T^{n}(x)-T^{n}(y)\right\| \leq k_{n}\|x-y\| \quad \text { for } x, y \in C \text { and } n=1,2, \ldots .
$$

We will consider in our main results mappings satisfying weaker assumptions. In 1972, Goebel and Kirk 5 proved that every asymptotically nonexpansive mapping $T: C \rightarrow C$ has a fixed point when $X$ is uniformly convex. Some generalizations of this result were proved by $\mathrm{Yu}$ and Dai [16] when $X$ is 2-uniformly rotund, by Martínez Yañez [12] and Xu [14] when $X$ is $k$-uniformly rotund for some $k \geq 1$, by Xu 15] when $X$ is nearly uniformly convex, by Lin, Tan and Xu [1] when $X$ satisfies the uniform Opial condition and recently by Kim and $\mathrm{Xu}[8]$ when $X$ has

Received by the editors April 10, 2000.

2000 Mathematics Subject Classification. Primary 47H09, 47H10.

Key words and phrases. Nonexpansive mapping, asymptotically nonexpansive mapping, retraction, common fixed points, convergence of iterates.

This research is partially supported by D.G.I.C.Y.T. PB 96-1338-C01-C02 and J.A. FQM 0127. 
uniform normal structure. From our results in Section 3, it can be derived that in all these cases the set of fixed points of $T$ is a nonexpansive retract of $C$. In some sense the nonexpansiveness of the retraction can be surprising because $T$ does not need to satisfy this property. The existence of such a nonexpansive retraction lets us use nonexpansive mappings techniques to prove the existence of a common fixed point for commuting families of asymptotically nonexpansive mappings. The possibility of obtaining common fixed point is noteworthy because the literature about such results for asymptotically nonexpansive mappings is quite rare (see [6] Theorem 2.1] for some results in this direction). Furthermore, we prove that the retraction $R$ onto the fixed point set satisfies some additional properties (i.e. $R$ is a $T$-ergodic retraction according to Bruck's notation [3]). This property will be used in the last section to prove (following some ideas in [9]) the convergence of iterates to a fixed point under suitable assumptions.

\section{Preliminaries}

In the following $X$ will be a Banach space with norm $\|\cdot\|$ and $C$ a nonempty weakly compact convex subset of $X$. We shall write $x=w-\lim _{n} x_{n}$ when the sequence $\left\{x_{n}\right\}$ converges weakly to $x$. We recall some definitions about properties satisfied by $X$ :

Definition 1. (a) $X$ is said to be nearly uniformly convex if it is reflexive and its norm is uniformly Kadec-Klee, that is, for any positive number $\epsilon$ there exists a corresponding number $\delta=\delta(\epsilon)>0$ such that

$$
\left.\begin{array}{c}
\left\|x_{n}\right\| \leq 1 \quad n=1,2, \ldots \\
w-\lim _{n} x_{n}=x \\
=\inf \left\{\left\|x_{n}-x_{m}\right\|: n \neq m\right\} \geq \epsilon
\end{array}\right\} \Longrightarrow\|x\| \leq 1-\delta .
$$

(b) $X$ is said to be uniformly convex in every direction if $\delta_{z}(\epsilon)>0$ for all $\epsilon>0$ and $z \in X$ with $\|z\|=1$, where $\delta_{z}(\epsilon)$ is the modulus of convexity of $X$ in the direction $z$ defined by

$$
\delta_{z}(\epsilon)=\inf \left\{1-\frac{1}{2}\|x+y\|:\|x\| \leq 1,\|y\| \leq 1, x-y=\epsilon z\right\} .
$$

(c) $X$ is said to have uniformly normal structure if $N(X)>1$, where $N(X)$ is the normal structure coefficient of $X$ defined by

$$
N(X)=\inf \left\{\frac{\operatorname{diam}(A)}{r(A)}: A \subset X \text { convex closed and bounded, } \operatorname{diam}(A)>0\right\}
$$

(here $\operatorname{diam}(A)$ is the diameter of $A$, i.e. $\sup \{\|x-y\|: x, y \in A\}$ and $r(A)$ is the Chebyshev radius of $A$, i.e. $\inf \{\sup \{\|x-y\|: y \in A\}: x \in A\})$.

(d) $X$ is said to satisfy the Generalized Gossez-Lami Dozo Property (GGLD) if

$$
\liminf _{n}\left\|x_{n}-x\right\|<\underset{m}{\limsup } \limsup _{n}\left\|x_{n}-x_{m}\right\|,
$$

whenever $\left\{x_{n}\right\}$ converges weakly to $x$ and $\left\{x_{n}\right\}$ is not norm-convergent.

For a mapping $T: C \rightarrow C$ we shall use the following definitions:

Definition 2. (a) $T$ is said to be asymptotically nonexpansive if there exists a sequence $\left\{k_{n}\right\}$ of real numbers with $\lim _{n} k_{n}=1$ such that

$$
\left\|T^{n}(x)-T^{n}(y)\right\| \leq k_{n}\|x-y\| \quad \text { for } x, y \in C \text { and } n=1,2, \ldots .
$$


(b) $T$ is said to be of asymptotically nonexpansive type if $T^{N}$ is continuous for some integer $N \geq 1$ and, for each $x \in C$, one has

$$
\limsup _{n}\left(\sup \left\{\left\|T^{n}(x)-T^{n}(y)\right\|-\|x-y\|: y \in C\right\}\right) \leq 0 .
$$

In [15] it is proved that $T$ has a fixed point if $X$ is nearly uniform convex and $T$ is of asymptotically nonexpansive type, while in [11] (resp. [8]) this result is proved when $X$ satisfies the uniform Opial condition (resp. when $X$ has uniform normal structure) and $T$ is asymptotically nonexpansive. In 8 it is also proved that there exists a fixed point if $X$ is UCED and has the GGLD property when $T$ is of asymptotically nonexpansive type. In fact, looking in detail at these proofs it is possible to get a fixed point in a slightly more general setting: We say that a nonempty closed convex subset $D$ of $C$ satisfies property $(\omega)$ with respect to $T$ if $\omega_{T}(x) \subset D$ for every $x \in D$ where

$$
\omega_{T}(x)=\left\{y \in C: y=w-\lim _{k} T^{n_{k}}(x) \text { for some } n_{k} \rightarrow \infty\right\} .
$$

(Obviously, $C$ itself verifies $(\omega)$.)

In connection with this fact we give the definition:

Definition 3. A mapping $T: C \rightarrow C$ is said to satisfy the ( $\omega)$-fixed point property $((\omega)$-fpp) if $T$ has a fixed point in every nonempty closed convex subset $D$ of $C$ which satisfies $(\omega)$.

The proofs of the results mentioned above lead to the following result:

Theorem 1 ([8], 11], [15]). Assume one of the following assumptions is satisfied:

(a) $T: C \rightarrow C$ is of asymptotically nonexpansive type and either $X$ is UCED and satisfies the GGLD property or $X$ is NUC.

(b) $T: C \rightarrow C$ is asymptotically nonexpansive and either $X$ satisfies the uniform Opial condition or $X$ has uniform normal structure.

Then $T$ satisfies the $(\omega)$-fixed point property.

In our main result we use another weaker definition of "asymptotic nonexpansiveness": $T$ is said to be weakly asymptotically nonexpansive if it satisfies the condition

$$
\limsup _{n}\left\|T^{n}(x)-T^{n}(y)\right\| \leq\|x-y\| \quad \text { for each } x, y \in C .
$$

Even though this definition could have rare interest from the point of view of fixed point theory (Tingley [13] has constructed an example of a bounded closed convex $C$ in Hilbert space and a continuous but fixed-point free $T: C \rightarrow C$ which satisfies $\lim _{n}\left\|T^{n}(x)-T^{n}(y)\right\|=0$ ), we shall prove some of our results for this class of mappings, because in this way we include different types of more suitable mappings.

We recall some other definitions: The set $C$ has the fixed point property for nonexpansive mappings (FPP) if every nonexpansive mapping $T: C \rightarrow C$ has a fixed point; $C$ has the conditional fixed point property for nonexpansive mappings (CFPP) [2] if either every nonexpansive $T: C \rightarrow C$ has no fixed points in $C$, or $T$ has a fixed point in every nonempty bounded closed convex $T$-invariant subset of C. 


\section{FIXED POINT SETS AS NONEXPANSIVE RETRACTS}

We state a theorem about the structure of the fixed-point set for asymptotically nonexpansive mappings:

Theorem 2. Let $X$ be a Banach space, $C$ a nonempty weakly compact convex subset of $X$, and $T: C \rightarrow C$ a weakly asymptotically nonexpansive mapping satisfying ( $\omega)$-fpp. Then, there exists a nonexpansive retraction $R$ from $C$ onto $F i x(T)$ which satisfies:

(i) $R \circ T=R$

(ii) every closed convex $T$-invariant subset of $C$ is also $R$-invariant.

Proof. Consider $C^{C}$ with the product topology induced by the weak topology on $C$. Then by Tychonoff's Theorem $C^{C}$ is compact. Now, define

$$
\begin{aligned}
\mathcal{N}:=\left\{f \in C^{C}\right. & : f \text { nonexpansive, } f \circ T=f, \text { and every closed convex } \\
& T \text {-invariant subset of } C \text { is also } f \text {-invariant }\} .
\end{aligned}
$$

Clearly $\mathcal{N}$ is a convex subset of $C^{C}$ and by using the weak lower semicontinuity of the norm, it is easy to check that $\mathcal{N}$ is closed in $C^{C}$. Hence $\mathcal{N}$ is a compact set. Furthermore $\mathcal{N} \neq \emptyset$. Indeed, consider the mappings $S_{n}=\frac{I+T+\ldots+T^{n-1}}{n}$. The sequence $\left\{S_{n}\right\}_{n \geq 1}$ satisfies $S_{n} \circ T-S_{n} \rightarrow 0$ as $n \rightarrow \infty$ on $C$ and it has a (pointwise weakly) convergent subnet $\left\{S_{n(\eta)}\right\}_{\eta}$. Define for each $x \in C S(x)=w-\lim _{\eta} S_{n(\eta)}(x)$ for every $x \in C$. We will check that $S \in \mathcal{N}$. Fix $x, y \in C$ and let $\epsilon$ be an arbitrary positive number. Since $T$ is weakly asymptotically nonexpansive, we can find an integer $k_{0} \geq 1$ (depending on $x$ and $y$ ) such that $\left\|T^{k}(x)-T^{k}(y)\right\| \leq(1+\epsilon)\|x-y\|$ for every $k \geq k_{0}$. Moreover, for $n_{0}\left(k_{0}\right) \in \mathbb{N}$ large enough we have

$$
\sum_{k=0}^{k_{0}-1} \frac{\left\|T^{k}(x)-T^{k}(y)\right\|}{n} \leq \epsilon\|x-y\| \quad \forall n \geq n_{0}\left(k_{0}\right) .
$$

Therefore

$$
\left\|S_{n}(x)-S_{n}(y)\right\| \leq(1+2 \epsilon)\|x-y\| \quad \forall n \geq n_{0}\left(k_{0}\right),
$$

and $\lim \sup _{n}\left\|S_{n}(x)-S_{n}(y)\right\| \leq\|x-y\|$. Hence,

$$
\begin{aligned}
\|S(x)-S(y)\| & \leq \liminf _{\eta}\left\|S_{n(\eta)}(x)-S_{n(\eta)}(y)\right\| \leq \limsup _{\eta}\left\|S_{n(\eta)}(x)-S_{n(\eta)}(y)\right\| \\
& \leq \limsup _{n}\left\|S_{n}(x)-S_{n}(y)\right\| \leq\|x-y\| .
\end{aligned}
$$

Moreover, $S(T(x))=w-\lim _{\eta} S_{n(\eta)}(T(x))=w-\lim _{\eta} S_{n(\eta)}(x)=S(x)$, because $S_{n} \circ T-S_{n} \rightarrow 0$ as $n \rightarrow \infty$ on $C$. Finally, if $D$ is a weakly compact convex $T$-invariant subset of $C$, by convexity it is clear that $D$ is $S_{n}$-invariant and thus $S$-invariant.

Defining a preorder in $\mathcal{N}$ by $f \preceq g$ if $\|f(x)-f(y)\| \leq\|g(x)-g(y)\|$ for every $x, y \in C$ and using the Bruck's method [1] we obtain a minimal element $R \in \mathcal{N}$ in the following sense:

$$
\begin{gathered}
\text { if } f \in \mathcal{N} \text { and }\|f(x)-f(y)\| \leq\|R(x)-R(y)\| \quad \forall x, y \in C, \\
\text { then } \quad\|f(x)-f(y)\|=\|R(x)-R(y)\| .
\end{gathered}
$$

For a given $x \in C$, consider the set $\mathcal{S}(R(x))=\{f(R(x)): f \in \mathcal{N}\}$. Then $\mathcal{S}(R(x))$ is a nonempty, convex (because $\mathcal{N}$ is convex) and closed subset of $C$ (notice that 
$\mathcal{S}(R(x))$ is the image of the set $\mathcal{N}$ under the projection $f \rightarrow f(R(x))$ of $C^{C}$ into $C)$.

We will show that $\mathcal{S}(R(x))$ satisfies $(\omega)$. Take $y \in \mathcal{S}(R(x))$ and $z \in C$ such that $T^{n_{k}}(y) \rightarrow z$ for some $n_{k} \rightarrow \infty$. There exists $f \in \mathcal{N}$ such that $y=f(R(x))$. Consider a subnet $\left\{T^{n_{k}(\eta)}\right\}$ of $\left\{T^{n_{k}}\right\}$ such that $s(x)=w-\lim _{\eta} T^{n_{k}(\eta)}(x)$ exists for every $x \in C$. We have $z=s(f(R(x)))$. Since $s$ is nonexpansive, $f \in \mathcal{N}$ and $s \circ f \circ T=s \circ f$, it follows that $s \circ f \in \mathcal{N}$ and then $z \in \mathcal{S}(R(x))$. Thus $\mathcal{S}(R(x))$ satisfies $(\omega)$ and $T$ has a fixed point in $\mathcal{S}(R(x))$, i.e. there exists $h \in \mathcal{N}$ such that $T(h(R(x)))=h(R(x))$. We shall prove that $R$ is a nonexpansive retraction from $C$ onto Fix $(T)$. Since any fixed point $p$ of $T$ is a closed convex $T$-invariant set we know that $R(p)=p$. Thus, it suffices to show that $R(p) \in$ Fix $(T)$ for each $p \in C$. Let $p \in C$ and $h \in \mathcal{N}$ be such that $T(h(R(p)))=h(R(p))$. Since $h \circ R \in \mathcal{N}$, we have:

$$
\|h(R(x))-h(R(y))\|=\|R(x)-R(y)\| \quad \forall x, y \in C .
$$

Let $y=h(R(p))$. We have $\|h(R(p))-h(R(y))\|=\|R(p)-R(y)\|$. Since $y \in F i x(T)$, we know that $y=h(R(y))=h(R(p))$. Thus $R(p)=R(y)$. Finally $R(y)=y$ and thus $R(p)=y \in \operatorname{Fix}(T)$. Clearly $R$ is a nonexpansive retraction from $C$ onto Fix $(T)$ and satisfies (i) and (ii) by the definition of $\mathcal{N}$.

Following the terminology introduced by Bruck in [3] we shall refer to a nonexpansive retraction from $C$ onto $F i x(T)$ which satisfies (i) and (ii) as a $T$-ergodic retraction.

Corollary 1. Let $X$ be a Banach space, $C$ a nonempty weakly compact and convex subset of $X$, and $T: C \rightarrow C$ a mapping of asymptotically nonexpansive type. Assume that one of the following conditions is satisfied:

(1) $X$ is nearly uniform convex.

(2) $X$ satisfies the uniform Opial condition and $T$ is asymptotically nonexpansive.

(3) $X$ has uniform normal structure and $T$ is asymptotically nonexpansive.

(4) $X$ is UCED with the GGLD property.

Then there exists a $T$-ergodic retraction $R$ from $C$ onto $F i x(T)$.

Remark 1. If, in addition, $T$ is weakly asymptotically regular, (2) in Corollary 1 can be derived from [4, Theorem 2].

In the following theorem we will show that a $T$-ergodic retraction can be obtained under some other assumptions. A mapping $T: C \rightarrow C$ is said to be asymptotically regular (respectively weakly asymptotically regular) if $\lim _{n}\left\|T^{n}(x)-T^{n+1}(x)\right\|=0$ for all $x \in C$ (resp. $\left.w-\lim _{n} T^{n}(x)-T^{n+1}(x)=0\right)$.

Theorem 3. Let $X$ be a Banach space which has the GGLD property and $C$ a nonempty weakly compact convex subset of $X$. Suppose $T: C \rightarrow C$ is a mapping satisfying one of the following conditions:

(a) $T$ is asymptotically regular (not necessarily continuous) and $\liminf _{n}\left|T^{n}\right|=1$.

(b) $T$ is weakly asymptotically regular and of asymptotically nonexpansive type. Then Fix $(T)$ is nonempty and there exists a T-ergodic retraction from $C$ onto $\operatorname{Fix}(T)$.

Proof. Case (a): Choose a subsequence $n_{k}$ of positive integers such that $\lim _{k}\left|T^{n_{k}}\right|=1$. Now, let $\left\{T^{n_{k}(\eta)}\right\}$ be a (pointwise weakly) convergent subnet of 
$\left\{T^{n_{k}}\right\}$, and define for each $x \in C$

$$
f(x)=w-\lim _{\eta} T^{n_{k}(\eta)}(x) .
$$

This mapping is nonexpansive by the weak lower semicontinuity of the norm. Furthermore $f$ satisfies:

$$
f(T(x))=w-\liminf _{\eta} T^{n_{k}(\eta)}(T x)=w-\liminf _{\eta} T^{n_{k}(\eta)}(x)=f(x) \quad \forall x \in C,
$$

because $T^{n}(x)-T^{n+1}(x) \rightarrow 0$ (note that we only need $T^{n}(x)-T^{n+1}(x) \rightarrow 0$ ). Therefore $f \circ T=f$. Since $X$ has the fixed point property for nonexpansive mappings (see [7]), there exists an $f$-ergodic retraction $R$ from $C$ onto Fix $(f)$. We shall prove that $\operatorname{Fix}(f)=\operatorname{Fix}(T)$.

Let $x \in F i x(f)$. Since $f(x)=x$ is a cluster point of $\left\{T^{n_{k}}(x)\right\}$ in the weak topology, there exists a subsequence $n_{k_{j}} \rightarrow \infty$ of positive integers in $\left\{n_{k}\right\}$ such that $T^{n_{k_{j}}}(x) \rightarrow x$. We claim that $T^{n_{k_{j}}}(x) \rightarrow x$. Otherwise, by the GGLD property we obtain:

$$
\begin{gathered}
\limsup _{j}\left\|T^{n_{k_{j}}}(x)-x\right\|<\underset{i}{\limsup } \limsup _{j}\left\|T^{n_{k_{i}}}(x)-T^{n_{k_{j}}}(x)\right\| \\
\leq \limsup _{i} \limsup _{j}\left|T^{n_{k_{i}}}\right|\left\|x-T^{n_{k_{j}}-n_{k_{i}}}(x)\right\| .
\end{gathered}
$$

On the other hand, the asymptotic regularity of $T$ implies

$$
\limsup _{j}\left\|x-T^{n_{k_{j}}-n_{k_{i}}}(x)\right\|=\limsup _{j}\left\|x-T^{n_{k_{j}}}(x)\right\| .
$$

Thus, limsup $\left\|T^{n_{k_{j}}}(x)-x\right\|<\lim \sup \left\|x-T^{n_{k_{j}}}(x)\right\|$ and this contradiction implies $T^{n_{k_{j}}}(x) \stackrel{j}{\rightarrow} x$. Finally we will prove that $T x=x$. Indeed, using the continuity of $T^{n_{l}}$, we have that $T^{n_{k_{j}}+n_{l}}(x) \rightarrow T^{n_{l}}(x)$ as $n_{k_{j}} \rightarrow \infty$. Moreover, the asymptotic regularity of $T$ implies that this sequence also converges to $x$. By induction, we can deduce that $T^{n_{l} s}(x)=x$ for $s=1,2 \ldots$. Therefore

$$
\|T(x)-x\|=\left\|T^{n_{l} s+1}(x)-T^{n_{l} s}(x)\right\| \rightarrow 0, \quad \text { as } s \rightarrow \infty .
$$

Thus $x$ is a fixed point for $T$ in $C$.

We have proved that Fix $(f) \subseteq$ Fix $(T)$. On the other hand, it is clear that Fix $(T) \subseteq$ Fix $(f)$ implying that $R$ is a nonexpansive retraction from $C$ onto Fix $(T)$. Because $f \circ T=f$, we have $R \circ T=R \circ f \circ T=R \circ f=R$. To complete the proof, it remains to check that $R$ satisfies (ii), but the definition of $f$ implies that every closed convex $T$-invariant subset of $C$ is $f$-invariant and so is $R$-invariant (recall that $R$ is an $f$-retraction).

Case (b): Using the whole sequence $\left\{T^{n}\right\}$ we proceed exactly as above, obtaining an $f$-ergodic retraction $R$ from $C$ onto Fix $(f)$. We claim that, again, Fix $(f) \subseteq$ Fix $(T)$. Indeed, if $x \in F i x(f)$, we can obtain a subsequence $\left\{T^{n_{k}}(x)\right\}$ of $\left\{T^{n}(x)\right\}$ such that $T^{n_{k}}(x) \rightarrow x$. Using the GGLD property if $T^{n_{k}}(x) \nrightarrow x$, we obtain

$$
B=\limsup _{j}\left\|T^{n_{j}}(x)-x\right\|<\limsup _{i} \limsup _{j}\left\|T^{n_{i}}(x)-T^{n_{j}}(x)\right\| .
$$

We can choose $\epsilon>0$ such that

$$
B+\epsilon<\limsup _{i} \limsup _{j}\left\|T^{n_{i}}(x)-T^{n_{j}}(x)\right\| .
$$


Since $T$ is of asymptotically nonexpansive type, there exists $n_{0}$ such that

$$
\left\|T^{n}(x)-T^{n}(y)\right\| \leq\|x-y\|+\frac{\epsilon}{4} \quad \text { for all } n \geq n_{0} \text { and all } y \in C .
$$

Select $n_{i_{0}}>n_{0}$ and a sequence $n_{i_{0}}+n_{0}<n_{j_{1}}<n_{j_{2}}<\ldots$ such that

$$
B+\epsilon \leq\left\|T^{n_{i_{0}}}(x)-T^{n_{j_{l}}}(x)\right\|=\left\|T^{n_{i_{0}}}(x)-T^{n_{i_{0}}+n_{j_{l}}-n_{i_{0}}}(x)\right\| .
$$

Taking $y=T^{n_{j_{l}}-n_{i_{0}}}(x)$ and $n=n_{i_{0}}$ in (1) we obtain

$$
B+\epsilon \leq\left\|x-T^{n_{j_{l}}-n_{i_{0}}}(x)\right\|+\frac{\epsilon}{4} \quad \text { for } l=1,2, \ldots
$$

For any fixed $l$, the weak asymptotic regularity of $T$ assures that $T^{n_{j_{l}}-n_{i_{0}}+n_{k}}(x)$ $\rightarrow x$ as $n_{k} \rightarrow \infty$ and we have

$$
\begin{aligned}
B+\epsilon \leq\left\|x-T^{n_{j_{l}}-n_{i_{0}}}(x)\right\|+\frac{\epsilon}{4} & \leq \limsup _{k}\left\|T^{n_{j_{l}}-n_{i_{0}}+n_{k}}(x)-T^{n_{j_{l}}-n_{i_{0}}}(x)\right\|+\frac{\epsilon}{4} \\
& \leq \limsup _{k}\left\|T^{n_{k}}(x)-x\right\|+\frac{\epsilon}{2}=B+\frac{\epsilon}{2},
\end{aligned}
$$

which is a contradiction. Thus $T^{n_{k}}(x) \rightarrow x$. Since $T^{N}$ is continuous and $T$ is weakly asymptotically regular it follows that $T^{N}(x)=x$. Hence $T^{N m}(x)=x$, for all $m$ and again the weak asymptotic regularity of $T$ implies that $T(x)=x$.

Remark 2. It is known that $X$ satisfies the GGLD property if either $X$ is UKK or $X$ satisfies the uniform Opial condition. Under these stronger assumptions, Theorem 3 (b) can be derived from results in [4] and [10].

\section{Common fixed point Sets for COMmuting families AND CONVERGENCE OF ITERATES}

Theorem 4. Let $X$ be a Banach space and $C$ a nonempty weakly compact convex subset of $X$. Assume that every asymptotically nonexpansive self-mapping of $C$ satisfies the $(\omega)-f p p$. Then for any commuting family $\mathcal{G}$ of asymptotically nonexpansive self-mappings of $C$, the common fixed point set of $\mathcal{G}$ is a nonempty nonexpansive retract of $C$.

In particular, the result holds under the hypotheses of Corollary 1 on $X$.

Proof. First we shall show that if card $\mathcal{G}=n, n=1,2, \ldots$, the common fixed point set of $\mathcal{G}$ is a nonempty nonexpansive retract of $C$. The proof is by induction on $n$.

If card $\mathcal{G}=1$, the result follows from Theorem 2 .

Now suppose the conclusion is true for card $\mathcal{G}=n-1$ and let $\mathcal{G}=\left\{T_{1}, T_{2}, \ldots, T_{n}\right\}$ be a commuting family of asymptotically nonexpansive self-mappings of $C$. Consider the family $\left\{T_{1} \circ T_{i}: i=2, \ldots, n\right\}$. Since it has $n-1$ commuting asymptotically nonexpansive self-mappings of $C$, the induction assumption implies that there exists a nonexpansive retraction $R$ of $C$ onto $\bigcap_{i=2}^{n} F i x\left(T_{1} \circ T_{i}\right)$. Moreover, $\bigcap_{i=2}^{n} \operatorname{Fix}\left(T_{1} \circ T_{i}\right)$ is a nonempty $T_{1}$-invariant set. Furthermore $T_{1} \circ R: C \rightarrow$ $\bigcap_{i=2}^{n} F i x\left(T_{1} \circ T_{i}\right)$ is a nonexpansive mapping. Indeed,

$$
T_{1} \circ R=T_{1} \circ\left(T_{1} \circ T_{2}\right)^{k} \circ R=T_{1}^{k+1} \circ T_{2}^{k} \circ R,
$$

and for all $x, y \in C$ we have

$$
\left\|T_{1} \circ R(x)-T_{1} \circ R(y)\right\| \leq\left|T_{1}^{k+1}\right|\left|T_{2}^{k}\right|\|x-y\| .
$$


Taking the limit as $k \rightarrow \infty$ we have that $T_{1} \circ R$ is nonexpansive. By Theorem 2 , $F i x\left(T_{1} \circ R\right)$ is a nonempty nonexpansive retract of $C$. If $x \in F i x\left(T_{1} \circ R\right)$ we have that $x \in \bigcap_{i=2}^{n} F i x\left(T_{1} \circ T_{i}\right)$ and $R(x)=x$. Thus $x \in$ Fix $\left(T_{1}\right)$ and $F i x\left(T_{1} \circ R\right) \subseteq$ $\bigcap_{i=2}^{n} \operatorname{Fix}\left(T_{1} \circ T_{i}\right) \cap$ Fix $\left(T_{1}\right)$, which clearly implies that $\operatorname{Fix}\left(T_{1} \circ R\right) \subseteq \bigcap_{i=1}^{n}$ Fix $\left(T_{i}\right)$.

On the other hand, $\bigcap_{i=1}^{n}$ Fix $\left(T_{i}\right) \subseteq$ Fix $\left(T_{1} \circ R\right)$. Indeed, if $x \in \bigcap_{i=1}^{n}$ Fix $\left(T_{i}\right)$, we have that $x \in \bigcap_{i=2}^{n} \operatorname{Fix}\left(T_{1} \circ T_{i}\right)$. Hence $R(x)=x$ and $x \in F i x\left(T_{1} \circ R\right)$. Therefore $\operatorname{Fix}\left(T_{1} \circ R\right)=\bigcap_{i=1}^{n}$ Fix $\left(T_{i}\right)$ which completes the proof in the finite case.

Now let $\mathcal{F}$ be the family of all finite intersections of the fixed-point sets of mappings in the commutative family $\mathcal{G}$. We have just proved that $\mathcal{F}$ is a family of nonexpansive retracts of $C$ and clearly this family is directed by set inclusion. Since $C$ has the FPP and the CFPP the result is an immediate consequence of the following lemma:

Lemma 1 ([2]). Suppose $C$ is a weakly compact convex subset of a Banach space, and has both the FPP and the CFPP. Then for any family $\mathcal{F}$ of nonempty nonexpansive retracts of $C$ which is directed by set inclusion, $H=\bigcap\{F: F \in \mathcal{F}\}$ is a nonempty nonexpansive retract of $C$.

Corollary 2. Let $X$ be a Banach space and $C$ a nonempty weakly compact convex subset of $X$. Assume that every asymptotically nonexpansive self-mapping of $C$ satisfies the $(\omega)$-fpp. If $T: C \rightarrow C$ is an eventually asymptotically nonexpansive mapping (i.e. there exists an integer $N \geq 1$ such that

$$
\left\|T^{i}(x)-T^{i}(y)\right\| \leq k_{i}\|x-y\|, \quad i \geq N,
$$

where $\left\{k_{i}\right\}$ is a sequence of real numbers with $\left.\lim _{i} k_{i}=1\right)$, then $T$ has a fixed point. Moreover, Fix $(T)$ is a nonexpansive retract of $C$.

Proof. It is easy to check that for an arbitrary self-mapping $f$ defined on a set $C$, one has Fix $(f)=F i x\left(f^{n}\right) \cap F i x\left(f^{n+1}\right)$. Under the hypotheses of Corollary 2, Fix $\left(T^{N}\right)$ and Fix $\left(T^{N+1}\right)$ are nonempty sets. Since $T^{N}$ and $T^{N+1}$ are asymptotically nonexpansive, $\operatorname{Fix}(T)=F i x\left(T^{N}\right) \cap \operatorname{Fix}\left(T^{N+1}\right)$ is a nonempty nonexpansive retract of $C$.

Finally, we are going to prove a result involving assumption on the existence of a $T$-ergodic retraction which implies the convergence of iterates.

Proposition 1. Let $C$ be a nonempty weakly compact convex subset of a Banach space $X, T: C \rightarrow C$ a mapping with Fix $(T) \neq \emptyset$ and suppose $\operatorname{dist}\left(T^{n}(x)\right.$, Fix $\left.(T)\right)$ $\rightarrow 0$ as $n \rightarrow \infty$. If there exists a nonexpansive retraction $R: C \rightarrow$ Fix $(T)$ satisfying $R T=R$, then $T^{n}(x)$ converges to $R(x)$.

Proof. For a given $\epsilon>0$ we can find an integer $N \geq 1$ such that

$$
\operatorname{dist}\left(T^{n}(x), F i x(T)\right)<\epsilon \quad \forall n \geq N .
$$

Choose $p_{n} \in \operatorname{Fix}(T)$ for each $n \geq N$ such that $\left\|T^{n}(x)-p_{n}\right\|<\epsilon$. Then

$$
\left\|R(x)-T^{n}(x)\right\| \leq\left\|R(x)-p_{n}\right\|+\left\|p_{n}-T^{n}(x)\right\| .
$$

Moreover

$$
\left\|R(x)-p_{n}\right\|=\left\|R\left(T^{n}(x)\right)-R\left(p_{n}\right)\right\| \leq\left\|T^{n}(x)-p_{n}\right\|,
$$

which implies $\left\|R(x)-T^{n}(x)\right\| \leq 2\left\|T^{n}(x)-p_{n}\right\|<2 \epsilon$ and the proof is complete. 
Corollary 3. Let $X, C$ and $T$ be as in Theorem 3(a). If there exists a constant $c>0$ such that for each $x \in C,\|x-T(x)\| \geq \operatorname{cdist}(x, F i x(T))$, then $T^{n}(x)$ converges to $R(x)$.

Proof. By assumption we have

$$
\operatorname{cdist}\left(T^{n}(x), \operatorname{Fix}(T)\right) \leq\left\|T^{n}(x)-T^{n+1}(x)\right\| \quad \forall n \geq 1 .
$$

Hence $\operatorname{dist}\left(T^{n}(x), \operatorname{Fix}(T)\right) \rightarrow 0$ as $n \rightarrow \infty$ and the conclusion follows from Proposition 1.

\section{REFERENCES}

[1] R.E. Bruck. Properties of fixed-point sets of nonexpansive mappings in Banach spaces. Trans. Amer. Math. Soc. 179 (1973), 251-262. MR 48:2843

[2] R.E. Bruck. A common fixed point theorem for a commuting family of nonexpansive mappings. Pacific J. Math. 53 (1974), 59-71. MR 50:14387

[3] R.E. Bruck. Asymptotic behavior of nonexpansive mappings. Contemp. Math. 18 (1983), 1-47. MR 85d:47057

[4] R.E. Bruck, T. Kuczumow and S. Reich. Convergence of iterates of asymptotically nonexpansive mappings in Banach spaces with the uniform Opial property. Colloq. Math. 65 (1993), 169-179. MR 94h:47106

[5] K. Goebel and W.A. Kirk. A fixed point theorem for asymptotically nonexpansive mappings. Proc. Amer. Math. Soc. 35 (1972), 171-174. MR 45:7552

[6] K. Goebel, W.A. Kirk and R.L. Thele. Uniformly lipschitzian families of transformations in Banach spaces. Canad. J. Math. 26 (1974), 1245-1256. MR 50:10919

[7] A. Jimenez-Melado. Stability of weak normal structure in James quasi reflexive space. Bull. Austral. Math. Soc. 46 (1992), 367-372. MR 93m:46011

[8] T.-H. Kim and H.-K. Xu. Remarks on asymptotically nonexpansive mappings. Nonlinear Analysis 41 (2000), 405-415. MR 2001b:47089

[9] W.A. Kirk and S.S. Shin. Fixed point theorems in hyperconvex spaces. Houston J. Math. 23 (1997), 175-188. MR 2000d:47080

[10] P.-K. Lin. Asymptotic behavior for asymptotically nonexpansive mappings. Nonlinear Analysis 26 (1996), 1137-1141. MR 97e:47092

[11] P.-K. Lin, K.-K. Tan and H.-K. Xu. Demiclosedness principle and asymptotic behavior for asymptotically nonexpansive mappings. Nonlinear Analysis 24 (1995), 929-946. MR 96a:47094

[12] C. Martínez-Yañez. A fixed point theorem on $k$-uniformly rotund spaces. Nonlinear Analysis 13 (1989), 857-861. MR 90g:47101

[13] D. Tingley. An asymptotically nonexpansive commutative semigroup with no fixed points. Proc. Amer. Math. Soc. 97 (1986), 107-113. MR 87g:47113

[14] H.-K. Xu. Existence and iterative convergence for fixed points of nonlinear mappings. Ph.D. Thesis, (1988), Xi'an Jiaotong University. (In Chinese).

[15] H.-K. Xu. Existence and convergence for fixed points of mappings of asymptotically nonexpansive type. Nonlinear Analysis 16 (1991), 1139-1146. MR 92h:47089

[16] X.T. Yu and X. Dai. A fixed point theorem of asymptotically nonexpansive mappings. J. Math. (PRC) 6 (1986), 255-262. MR 87k:47135

Departamento de Análisis Matemático, Facultad de Matemáticas, Universidad de Sevilla, Apdo. 1160, Sevilla 41080, Spain

E-mail address: tomasd@cica.es

Departamento de Análisis Matemático, Facultad de Matemáticas, Universidad de Sevilla, Apdo. 1160, Sevilla 41080, Spain

E-mail address: ploren@cica.es 